\title{
DE LA HISTORIA ECLESIÁSTICA A LA HISTORIA RELIGIOSA. UNA TRAYECTORIA HISTORIOGRÁFICA
}

FROM THE ECLESIASTIC HISTORY TO RELIGIOUS HISTORY. A BIOGRAPHICAL TRAJECTORY

\author{
Feliciano Montero \\ Universidad de Alcalá de Henares
}

Entregado el 15-3-2015 y aceptado el 4-6-2015.

Resumen: Desde una perspectiva testimonial, el autor presenta un estado de la cuestión sobre la historia religiosa española, basada en su propia trayectoria historiográfica. Con el título de este trabajo, se pretende describir un proceso y un programa de renovación en relación a la historia de la Iglesia y del catolicismo español desde el conocimiento comparativo de otras realidades europeas.

Palabras clave: historiografía, historia religiosa, catolicismo, laicismo.

\begin{abstract}
From a testimonial perspective, the author presents a state of the investigation on the religious history in Spain, based on its own historiographical trajectory. The title of this work describes a process and a program of renewal in relation to the history of the Catholic Church and the Spanish Catholicism through the comparative knowledge of the other european realities.
\end{abstract}

Key words: historiography, religious history, catholicism, laicism. 
Con el título «de la historia eclesiástica a la historia religiosa»-que he utilizado ya en varias ocasiones en diferentes artículos y balances historiográficos - he querido describir un proceso pero, sobre todo, proponer a la historiografía española un camino y un programa de renovación en relación a la historia de la Iglesia y del catolicismo español. Eso sí, siguiendo los pasos de otras historiografías europeas cercanas, particularmente la francesa, que habían iniciado tempranamente esa renovación. Se me pidió, en una línea enfocada a través de un mirada testimonial y memorialística (lo que he intentado no sin cierto rubor frente a una tarea que se acerca a la egohistoria), que presentara un estado de la cuestión en el que apareciesen sucesivamente los impactos, las influencias y las relaciones con las historiografías francesa, italiana y portuguesa, al hilo de mi propia trayectoria y proyectos, sin olvidar el equipo y los colegas a cuyo lado se ha ido configurando este largo recorrido, y que ha tenido un hito historiográfico con la organización en abril de 2015 en la Universidad de Alcalá, y junto a Julio de la Cueva y Joseba Louzao, de un Encuentro Internacional de Historia Religiosa que pretendía ser un balance de lo acometido en estos primeros años del siglo $\mathrm{XXI}^{1}$.

\section{Una historia secular y laica. La historiografía francesa y su impacto en la española}

El punto de partida, siguiendo una larga tradición de sana dependencia intelectual (que para mi generación fue fundamentalmente de carácter francés), debo situarlo en el descubrimiento temprano de la historiografía francesa desarrollada entre los años setenta y ochenta del siglo XX, al hilo de mi temprana investigación sobre la historia del catolicismo social en la época de la publicación de la encíclica Rerum Novarum. El contacto indirecto, que más tarde se convirtió en directo y personal, con algunos de los principales representantes de la nueva historia religiosa (quiero recordar a Jean- Marie Mayeur, Yves- Marie Hilaire o Gérard Cholvy, entre otros muchos) me abrió un inmenso campo de objetos y objetivos de in-

\footnotetext{
${ }^{1}$ Algunas de estas reflexiones ya las he presentado en otros trabajos anteriores, especialmente en Feliciano Montero, «La historiografía española entre la historia eclesiástica y la religiosa», en B. Pellistrandi (ed.), Hacer la Historia del Siglo XX, Biblioteca Nueva, Madrid, 2003, pp. 266-281. Pido excusas, por tanto, por las posibles reiteraciones en las que pueda caer.
} 
vestigación, que, por otra parte, aparecía como una especie de traducción de la historia social de la corriente de Annales (que tanto había influido en mis últimos años de licenciatura) en el campo de la historia religiosa. Una nueva historia que, como ha confesado Hilaire, uno de sus principales mentores y practicantes era, a partir de completas historias diocesanas, una forma de practicar el ideal de historia social, entendida ésta como una «historia total», de la escuela de Annales. En efecto la investigación diocesanas de Hilaire sobre la diócesis de Arras, siguiendo este programa historiográfico, comenzaba por la geografía y la demografía de la diócesis, continuaba con el estudio institucional de la Iglesia en todos su niveles y categorías sociales posibles, y culminaba con el estudio de su proyecto pastoral restaurador, incluyendo el análisis de las «obras católicas», las devociones y los cultos ${ }^{2}$. El modelo fue desarrollado en multitud de investigaciones que cartografiaron la historia religiosa francesa. Por su parte, este proyecto de investigación era también la aplicación historiográfica del proyecto de sociología religiosa pastoral, iniciado por Gabriel Le Bras y continuado por su principal discípulo, el canónigo Fernand Boulard, en el contexto de la búsqueda de respuestas pastorales a la descristianización o lo que se popularizó como la «apostasía de las masas» ${ }^{3}$.

En un panorama español tan centrado, aún en la década de los setenta en el estudio de las relaciones Iglesia-Estado o, todo lo más, en el estudio de la conflictividad clericalismo-anticlericalismo, o del catolicismo político y social, el programa de investigación del grupo de historiadores franceses, que se concentraban en estudiar de forma interdisciplinar la historia religiosa moderna y contemporánea del país vecino era estimulante y provocador. El estado de la cuestión que presentaba el propio Jean- Marie

${ }^{2}$ Véase, para profundizar en estos aspectos, la entrevista de Hilaire, en J. Escudero Imbert, «Conversación en Pamplona con Yves Marie Hilaire», Anuario de Historia de la Iglesia, 7 (1998), pp. 303-319. Su investigación sobre la diócesis de Arras, Une Chrétienté au XIXe siècle?: La vie religieuse des populations du diocèse d'Arras (1840-1914), Université de Lille III, Villeneuve-d'Ascq, 1977

${ }^{3}$ La alerta del libro de Henri Godin e Yvan Daniel, La France: pays de mission?, Cerf, Paris, 1943, era paralela a la influencia del plan de estudio de Fernand Boulard, sobre la práctica religiosa en París, que los historiadores asumieron retrospectivamente en FrançoisAndré Isambert y Jean-Paul Terrenoire, Atlas de la pratique religieuse des catholiques en France, Presses de Sciences Po, Paris, 1980 y en la obra Matériaux pour l'histoire religieuse du peuple français, Presses de sciences Po/École des Hautes Études en Sciences Sociales/ Éditions du CNRS, Paris, t. 1 (1982), t. 2 (1987) y t. 3 (1992). 
Mayeur a la altura de 1975 agrupaba un amplio panorama de trabajo en los siguientes campos:

- Las iglesias como sociedades religiosas: instituciones y poderes, lugares de culto, ritos y obligaciones, o relaciones iglesias y Estado.

- La religión vivida: práctica religiosa, vida sacramental y fiestas litúrgicas; catecismo, predicación, misiones; devociones, obras y movimientos; permanencia y transformación de la religión popular.

- La vida intelectual y vida espiritual.

- Las iglesias en la Sociedad: catolicismo social; las actitudes políticas; y las misiones.

- Las iglesias contestadas, el anticlericalismo e irreligión: la contestación interna ${ }^{4}$.

Ahora bien, cómo podíamos llevar a cabo un programa similar para el caso español, si el estudio de los temas religiosos, en primer lugar, parecía reservado exclusivamente al interés y la competencia de los centros de estudios eclesiásticos y a los investigadores clericales. Solamente algunos pioneros, que actuaban como francotiradores historiográficos (principalmente puede señalarse la labor de José Manuel Cuenca Toribio ${ }^{5}$, luego José Andrés-Gallego ${ }^{6}$ ), se estaban ocupando del tema en el ámbito de las

${ }^{4}$ Jean- Marie Mayeur, L'Histoire religieuse de la France, 19 et 20 siecle. Problemes et Methodes, Beauchesne, Paris, 1975. Otra revisión historiográfica posterior de Bernard Plongeron, Religión et Societés en Occident, XVI-XX siecles. Recherches francaises et tendences internationales, C.N.R.S, Paris, 1982. hace un balance cualitativo y cuantitativo de la investigación francesa e internacional sobre historia religiosa. Este autor señalaba como nuevos centros de interés en el horizonte de los años ochenta: el discurso iconográfico; la conciencia misionera y la nueva historia de las misiones; y la relación de las Iglesias, el Estado y la cultura en el cambio social.

${ }^{5}$ Los estudios pioneros de José Manuel Cuenca Toribio sobre la Iglesia y la Jerarquía española en el siglo XIX, principalmente en la época de Isabel II y sobre el sexenio liberaldemocrático. Él también se inspiró y reforzó su línea de investigación en contacto con la historiografía francesa. Participa por ejemplo en el Coloquio organizado por L’Ecole Francaise a Rome sobre Pío X, Achille Ratti pape Pie XI. Actes du colloque de Rome (15-18 mars 1989), École Française de Rome, Roma, 1996. Su impulso a una nueva historiografía sobre la Iglesia y el catolicismo español se plasma principalmente en los Coloquios organizados en El Escorial, entre 1974 y 1978. Las ponencias de las Semanas se publicaron Aproximación a la Historia social de la Iglesia española contemporánea (1978), Estudios históricos sobre la Iglesia española contemporánea (1979) y La cuestión social en la Iglesia española contemporánea (1981).

6 La obra de José Andrés- Gallego, centrada sobre todo en la época de la Restauración, supone un replanteamiento nuevo del peso del catolicismo político y social (La política re- 
universidades civiles. Por otro lado, en los planes de estudio de la licenciatura de Historia, y sobre todo en el área de historia contemporánea, la referencia a temas de historia religiosa estaba ausente, salvo algunos estudios sobre la relación Iglesia-Estado, y sobre la conflictividad social y política generada por el factor católico; pero además esto último tratado más bien desde los prejuicios prolongados de la «cuestión religiosa» como cuestión histórica pendiente.

La nueva historia religiosa francesa, a la que algunos mirábamos con sana envidia, era, sin duda, el fruto de un largo proceso de secularización, que también había afectado al estudio y la investigación del hecho religioso en sus diversas expresiones. La historiografía católica francesa llevaba tiempo reflexionando sobre el proceso de confrontación catolicismolaicismo desde la Revolución francesa, pasando por la ley de separación de 1905. Pero la nueva historia religiosa iba más allá de la historia política. Trataba de llevar a cabo, en su propia parcela, el programa de la «historia total» de Annales, tal como lo expresa Hilaire retrospectivamente: «Al comenzar mi tesis doctoral, y siguiendo la invitación de mis maestros, dirigí mis intereses hacia la historia global y no sólo hacia la historia religiosa $\gg^{7}$.

Un mero repaso a las noticias que ofrece el boletín del Grupo de investigación del CNRS (GRECO n. ${ }^{\circ}$ 2) centrado en la historia religiosa moderna y contemporánea, nos pone en contacto con los grupos de investigación (ubicados en diversas universidades francesas), y con los objetivos comunes y prioritarios de investigación, y los específicos de cada grupo tal como eran definidos en 1983:

- Investigaciones sobre la piedad popular.

- Transformaciones de la conciencia misionera cristiana en las épocas moderna y contemporánea.

- Permanencias, reformas, disidencias en el s. XVI.

ligiosa en España, 1889-1913, Editora Nacional, Madrid, 1975 y Pensamiento y acción social de la Iglesia en España, Espasa, Madrid, 1984). La inspiración y las relaciones con la historiografía francesa se aprecian en la tesis de su discípulo y estrecho colaborador, Antón Pazos, El clero navarro 1900-1936. Origen social, procedencia geográfica y formación sacerdotal, Ediciones Universidad de Navarra, Pamplona, 1990 y especialmente en la obra conjunta de síntesis sobre La Iglesia en la España contemporánea, Encuentro, Madrid, 1999, inicialmente preparada y publicada en francés por la editorial parisina Cerf, en una colección de Historias nacionales dirigida por el profesor Hilaire.

7 J. Escudero Imbert, «Conversación en Pamplona...», op. cit. 
- Organizaciones de juventudes judía y cristianas en Francia.

- Atlas religioso de la Francia moderna.

- La práctica pastoral de los obispos franceses ( a partir del análisis sistemático del «Repertorio de visitas pastorales»).

- Las cofradías: su devoción, su historia, su sociabilidad.

- El espacio y los sagrado: recensión de lugares de culto y de devociones.

- El clero secular y regular: mapas de implantación de órdenes y congregaciones.

- Diccionario biográfico del mundo religioso francés contemporáneo (1800-1962).

- Materiales para la historia religiosa del pueblo francés (18001939): continuando el trabajo emprendido por Fernand Boulard (como ya hemos señalado, el discípulo aventajado del sociólogo Le Bras) de cuantificar la práctica religiosa en París.

- Repertorio de visitas pastorales de Francia.

El temario y las secciones del Coloquio organizado por este Grupo de Investigación con motivo del bicentenario de la Revolución francesa confirmaba el programa de investigación: mutaciones revolucionarias y tradiciones confesionales en los países ocupados; la vida religiosa y sacramentalización de los fieles; resistencias y mutaciones culturales; la religión revolucionaria; Catequesis y Enseñanza; vida asociativa y cofradías; devociones y peregrinaciones; Iconografía judía y cristiana ${ }^{8}$.

Ciertamente la renovación historiográfica también se beneficiaba del clima mental conciliar y postconciliar de la teología del Vaticano II: la concepción de la Iglesia como «Pueblo de Dios» afectaba directamente al sujeto de la historia que ya no podía quedar reducido a la Jerarquía de la Iglesia, los clérigos, o los religiosos. El papel del laico en la Iglesia legitimaba la incursión de los historiadores laicos en el estudio histórico así como el análisis del papel de los laicos y de sus organizaciones como sujeto histórico. Por otra parte, la propuesta de una Iglesia abierta y tolerante, dialogante con el mundo moderno, que propone la Gaudium et Spes, impulsaba un enfoque historiográfico igualmente abierto, confrontado con los conceptos y los marcos de la historiografía civil, principalmente en relación con el conflicto Iglesia-modernidad y los procesos de seculariza-

${ }^{8}$ Me estoy refiriendo al Coloquio de Chantilly, celebrado bajo el título general Pratiques religieuses dans l'Europe revolutionnaire(1770-1820) en noviembre 1986. 
ción. Dentro de la Iglesia la historia debería ser una disciplina académica, diferente e independiente de la teología, sometida a las mismas reglas críticas de la historia académica ${ }^{9}$. La renovación historiográfica de la historia religiosa se recogía también en varios números y artículos de la revista de teología Concilium, creada dentro del impulso conciliar en 1965 por un grupo de reconocidos teólogos entre los que destacaban Yves Congar, Hans Küng, Johann-Baptist Metz, Karl Rahner o Edward Schillebeeckx, cuya lectura me influyó tempranamente ${ }^{10}$.

En relación con el estatuto y la identidad específica del historiador de lo religioso, Hilaire, tras señalar el predominio en Francia del historiador creyente (al menos en su generación del Greco 2), apelaba a una historia ni «apologética» ni «culpabilista», «sino una historia comprehensiva que rechazara especialmente el anacronismo». Una historia científica en diálogo con la comunidad académica, homologable al trabajo de otros colegas, aunque reconociendo una cierta especificidad en el estudio de algunos temas: «El historiador creyente tiene en cierto modo el mismo cometido, aunque sin disociarse de sus colegas, de dar otra tonalidad, una orientación que recuerde algunos aspectos que otros podrían descuidar. En particular debe evocar la presencia y la fecundidad de la santidad en la historia» ${ }^{11}$.

Este proceso de cambio historiográfico, que podemos calificar de secularización de la historia de la Iglesia y del catolicismo, es lo que mejor justifica la validez del título propuesto como hilo argumental: «De la historia eclesiástica a la historia religiosa». En el caso francés las raíces del cambio estaban en el largo proceso de adaptación (primero) y asunción (después) de la laicidad . Por otro lado, no es casual que algunos historiadores protagonistas del cambio (como el propio Hilaire, o René Remond) se hubieran educado en la mentalidad y el método de la Acción Católica especializada, en concreto la Juventud Estudiante Católica (JEC), -que anticipa el enfoque pastoral del Vaticano II - y que fueran activos agentes

9 Sobre la relación de la historia de la Iglesia con la teología, véase Evangelista Vilanova, «Historia y Teología», XX Siglos, 35 (1998), pp. 52-60.

${ }_{10}$ Entre ellos destaco el trabajo del jesuita italiano Giacomo Martina, «La contribución del liberalismo y del socialismo para una mejor autocomprensión de la Iglesia», Concilium, 67 (julio-agosto 1971), pp. 104-113. La propuesta de Martina de análisis de la evolución de la Iglesia y del catolicismo desde su confrontación-diálogo con el liberalismo y el socialismo se convirtió en buena medida en el hilo conductor de mis propios proyectos.

11 La entrevista, ya citada de Escudero Imbert (p. 310). 
de las actividades del Centro de Intelectuales católicos en los años $1950-70^{12}$.

La España católica, como se sabe (aunque se ha estudiado poco), se sintió especialmente desconcertada por la novedad del Concilio, pero algunos de los primeros impulsores del cambio historiográfico seguramente se inspiraron y confirmaron en ese contexto mental. En algunos ámbitos eclesiásticos y seglares, como desde luego la Acción Católica especializada, también se anticipó y vivió el Concilio y el postconcilio, y se abrió camino una renovación teológica y filosófica, y en alguna medida historiográfica. Pienso, por ejemplo en el impulso de la historia del catolicismo social. La visión autocrítica del pasado del catolicismo social (especialmente en el libro de Benavides sobre el canónigo asturiano Arboleya) era un reflejo y explicación autocrítica de ciertos defectos y retrasos del catolicismo español, necesaria en la coyuntura postconciliar y tardofranquista de la década de $1965-1975^{13}$. Mi propia investigación sobre la recepción de la Rerum Novarum se inscribe en esa onda, aunque estuviera más inspirada e influída por la buena y abundante historiografía francesa ${ }^{14}$.

12 La nueva orientación historiográfica era coherente con la mentalidad social y eclesial aprendida en la JEC, a partir de la pedagogía de la Revisión de Vida. Algunos trabajos de René Remond sobre la Acción Católica francesa de la postguerra se entrelazan con su propia implicación militante en las organizaciones. Sobre el Centro de Intelectuales católicos, Claire Toupin-Guyot, Les intellectuels catholiques dans la société française: le Centre catholique des intellectuels français (1941-1976), Presses Universitaires de Rennes, Rennes, 2002

13 Domingo Benavides, El fracaso del catolicismo social. Arboleya Martínez, 1870-1951, Nova Terra, Barcelona, 1973. Tuvo impacto y provocó cierta polémica, por su dependencia casi exclusiva del archivo Arboleya, y por la crítica directa y explícita a la orientación más paternalista y clerical del segundo Marques de Comillas y del jesuita P. Nevares. El mismo Benavides publicó enseguida una buena síntesis del catolicismo político y social durante la Restauración, Domingo Benavides, Democracia y Cristianismo en la España de la Restauración, edit. Nacional, Madrid, 1978. Un balance e interpretación de lo que supuso ese boom de los estudios sobre el catolicismo social en la España de la Transición en Feliciano Montero, «El catolicismo social en España. Balance historiográfico», en Benoit Pellistrandi, L'histoire religieuse en France et en Espagne, Casa de Velázquez, Madrid, 2004, pp. 389-409.

${ }^{14}$ Feliciano Montero, El primer catolicismo social y la Rerum Novarum en España, Centro Superior de Investigaciones Científicas, Madrid, 1983. En algunos apartados, en las notas y en la lista bibliográfica se pueden ver las lecturas de autores franceses, desde el protagonista La Tour du Pin al historiador más influyente Jean- Marie Mayeur, con un artículo tan reconocido sobre los orígenes intransigentes del catolicismo social, «Catholicisme intransigeant, catholicisme social, democratie chretienne», Annales ESC, 1972, pp. 482-489. 
También en esa onda historiográfica renovadora, ligada al tiempo de la Transición, habría que mencionar el proyecto colectivo dirigido por Miquel Batllori sobre «El anticlericalismo en España». No era casual que ese Proyecto de investigación se planteara por los mismos autores que, en ese momento, habían iniciado la edición crítica del Arxiu Vidal i Barraquer depositado en la Abadía de Montserrat ${ }^{15}$. Se trataba de un proyecto interdisciplinar que buscaba abordar la cuestión histórica en una perspectiva plural, dialogal y abierta. Aún inconcluso, dio lugar a algunas publicaciones notables como la síntesis del antropólogo Julio Caro Baroja, el estudio de Arbeloa sobre la encuesta socialista de finales del siglo XIX, o la aproximación de Díaz Mozaz desde la sociología religiosa ${ }^{16}$.

Un poco más tarde, a inicios de la década de los noventa, pero haciendo gala del mismo espíritu de la renovación historiográfica ligada a la Transición, Juan María Laboa, que era profesor de Historia de la Iglesia en la Universidad Pontificia de Comillas, fundó la revista $X X$ Siglos con la intención de divulgar con rigor la historia de la Iglesia y del catolicismo español. En todos los números de la revista hasta su final, que se produjo en el 2008, la historia del siglo Xx ocupó bastante espacio, dedicando números monográficos a recuperar la aportación de la Iglesia y los católicos al proceso de la Transición ${ }^{17}$. Algunos de esos trabajos siguen siendo aportaciones ineludibles a la hora de acercarse a aquel tiempo, ya que ofrecen muchas pistas que permitirán profundizar en el conocimiento de un período aún necesitado de investigación.

Pero, en ese momento, esa renovación historiográfica quedó preferentemente limitada o reducida al ámbito del catolicismo social y político. Apenas se planteaba una renovación global de la historia religiosa en la

15 Miquel Batllori y Víctor Manuel Arbeloa, Arxiu Vidal i Barraquer. L'Esglesia i Estat en la 2. ${ }^{a}$ República Española, Publicacions de la Abadía de Montserrat, 1974-1991 (en 7 volúmenes).

${ }^{16}$ La memoria y los trabajos parciales se pueden consultar en la Biblioteca de la Fundación March, que fue la institución financiadora del Proyecto. Además, Julio Caro Baroja, Introducción a una historia contemporánea del anticlericalismo, Istmo, Madrid, 1980; Víctor Manuel Arbeloa, Socialismo y anticlericalismo, Taurus, Madrid, 1973; José María Díaz Mozaz, Apuntes para una sociología del anticlericalismo español, Ariel, Barcelona, 1976.

17 «Los católicos en la lucha por la democracia», XX Siglos 16, 1993; «Los católicos y el nuevo Movimiento obrero», XX Siglos , 22, 1994; «Los Democrata Cristianos en la transición española», XX Siglos, 26 (1995); «La Acción Católica durante el franquismo», XX Siglos ,49 (2001) 
perspectiva social y cultural, «total», del grupo de historiadores franceses. En el caso español la pervivencia de la hegemonía católica, institucional y mental, había limitado el proceso de secularización, y por supuesto, su autoconciencia y estudio. Incluso la revisión autocrítica que la propia institución eclesiástica genera en el quinquenio final del franquismo, cuya expresión máxima, sería la ponencia primera de la Asamblea Conjunta de 1971, quedó reducida a un ámbito fundamentalmente intraeclesial; por más que fuera percibido y aprovechado agradecidamente por sectores de la sociedad civil y de la oposición antifranquista y provocara una importante crisis intraeclesial ${ }^{18}$.

Quizá eso explica que en el nuevo contexto político de la Transición y del reconocimiento amistoso de la separación Iglesia-Estado (la aconfesionalidad proclamada en la Constitución de 1978), la historia religiosa siguiera anclada en ámbitos eclesiásticos, salvo escasas excepciones. Y que, pasado el tiempo, se reprodujeran viejos prejuicios y conflictos políticos, obstáculos y frenos también en el tratamiento historiográfico de la cuestión religiosa, sobre todo en relación con la cuestión de la «persecución religiosa» y las violencias y represiones durante la guerra civil.

Por otro lado, en el ámbito académico, si bien los estudios sobre el catolicismo social y político tuvieron un cierto impacto y reconocimiento, y contribuyeron sin duda al aumento cuantitativo y cualitativo de las investigaciones, siguieron siendo temas relativamente marginales en el mundo académico. Habría que esperar aún algunos años para que se abriera camino, por ejemplo, un nuevo enfoque en el estudio del anticlericalismo con los estudios de Julio de la Cueva o Pilar Salomón ${ }^{19}$.

Sin embargo, más allá del estudio del catolicismo social y político, la influencia de la nueva historia religiosa francesa y la aspiración de hacer algo parecido en España no dejó de pesar e inspirar algunos estudios

18 Todos los trabajos y conclusiones de la Asamblea se pueden consultar en Asamblea Conjunta Obispos-Sacerdotes, Biblioteca de Autores Cristianos, Madrid, 1971.

19 Julio de la Cueva Merino, Clericales y anticlericales. El conflicto entre confesionalidad y secularización en Cantabria (1875-1923) Universidad de Cantabria, Santander, 1991; Pilar Salomón Cheliz, Anticlericalismo en Aragón. Protesta popular y movilización política (1900-1939), Prensa Universitaria de Zaragoza, Zaragoza, 2002. Véase el balance historiográfico de Julio de la Cueva y Feliciano Montero, «El impacto del hispanismo y de los estudios internacionales sobre catolicismo y secularización en la historiografía española», en Alfonso Botti, Marco Cipolloni y Vittorio Scotti Douglas (eds.), Ispanismo internazionale e circolazione delle storiografie negli anni della democrazia spagnola (19782008), Rubettino, Torino, 2014, pp. 565-597 
y objetivos: Principalmente la tesis de Anton Pazos sobre el clero navarro; algunos estudios diocesanos, vinculados a ciertos pontificados, como especialmente el de Costa y Borrás en Barcelona, el de Vilatmijana en la diócesis de Tortosa o el de de Morgades en la diócesis de Vich ${ }^{20}$.

La celebración del XVII Congreso Internacional de Ciencias históricas, celebrado en Madrid en 1990, y en concreto la organización de las sesiones de la Comisión de historia eclesiástica, fue también la ocasión para la confirmación del impacto de los estudios europeos sobre la secularización. La revista del CSIC Hispania Sacra, dirigida por J. Andrés Gallego, recogió las ponencias y comunicaciones de H. MacLeod, de W. Callaham y de Y. M. Hilaire entre otros, sobre urbanización, secularización y respuesta pastoral en los siglos XIX y XX ${ }^{21}$.

Quizá el planteamiento global que mejor refleja esa inspiración es la síntesis de historia de la Iglesia contemporánea escrita por J. Andrés Gallego y A. Pazos para la editora francesa Cerf. Especialmente el primer volumen de la edición española presenta, más que una síntesis, imposible, por la falta de investigaciones monográficas, todo un programa de estudio. En el apartado «La Iglesia concordataria», incluye los siguientes capítulos: los eclesiásticos (incluyendo el presupuesto, la formación en los seminarios, la extracción social, la proyección misionera extrapeninsular), la espiritualidad y la ética, el drama liberal(es decir los pleitos católicos con los liberales en relación con la enseñanza, la imprenta, el matrimonio), «los ejércitos» ( es decir el conjunto de asociaciones y obras que constituyen el llamado «Movimiento católico»), la sociología de la movilización(una valoración cualitativa, cuantitativa y territorial de la presencia, implantación y movilización clerical y seglar), el catolicismo de Trento (breve incursión en el «universo devocional»), y finalmente, «recristianizaciones, descristianización (estudio del grado de difusión y

20 Casimir Martí, L'esglèsia de Barcelona (1850-1857). Implantació social i dinamismes interns, Curial, Barcelona, 1984; Jordi Figuerola, El bisbe Morgades i la formació de l'Església catalana contemporània, Publicaciones de la Abadía de Montserrat, 1994; o Carmen Ibañez Gisbert, La diócesis de Tortosa en el pontificado de Monseñor Benito Vilamitjana i Vila (1862-1879), UNED, Tortosa, 1991.

${ }^{21}$ La publicación de estas ponencias en Hispania Sacra, 86 (1990), bajo el título «Las Iglesias en la ciudad. Los tiempos modernos»; Hugh McLeod, «Secularisation and revival in the 19th century city», pp. 391-404; Yves-Marie Hilaire, «Observations sur la pratique religieuse urbaine en France pendant la premiere moitié du XX siecle», pp. 457-467; y la de William Callahan, «Response to urbanization: Madrid and Barcelona, 1850-1930», pp. 445-451. 
eficacia del movimiento secularizador o anticlerical frente al movimiento

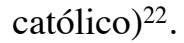

El balance comparado de la historia religiosa contemporánea en Francia y España organizado en la Casa de Velázquez en 2001 es el mejor retrato de la situación: de desigualdad por un lado, pero por otro, de inspiración e impulso renovador por parte de la historiografía española, que ha marcado la trayectoria de una nueva generación de historiadores españoles, mucho más liberados de los viejos prejuicios, y, sobre todo, más abiertos a influencias historiográficas no sólo francesas ${ }^{23}$.

\section{La historiografía italiana: la utilidad del concepto «movimiento católico»}

Si el contacto con la historiografía francesa me había permitido plantear el estudio del catolicismo social en una dimensión y perspectiva nueva, el conocimiento de la historiografía italiana sobre el Movimiento Católico (especialmente la consulta del Dizionario di Storia del Movimento Cattolico), y el encuentro simultáneo con la tesis innovadora de Pere Fullana sobre El Moviment Catolic a Mallorca (no casualmente iniciada en Roma) me abrió un nuevo horizonte. El estudio del catolicismo social debía ser integrado en un conjunto global, que reflejaba, en sus diversas dimensiones, actividades y «obras», la existencia de un proyecto total de recristianización o restauración social católica de la nueva sociedad liberal e industrial. Los Congresos Católicos nacionales e internacionales, entre ellos notablemente la serie de Opera di Congressi italianos, habían inspirado la serie de Congresos Católicos nacionales, celebrados en España entre 1889 y 1902 . Personalmente había utilizado ampliamente su sección de asuntos de caridad o asuntos sociales como hilo conductor para detectar el grado de recepción de la Rerum Novarum en España Pero lo que las amplias crónicas de los Congresos reflejaban era ese proyecto global de afrontar los distintos retos de la modernidad: la escuela, la prensa, la asistencia y la acción social; en fin, en general, la tendencia secularizadora de las ins-

22 José Andrés-Gallego y Antón Pazos, La Iglesia en la España contemporánea, 1. ${ }^{\circ}$, 1800-1936, Encuentro, Barcelona. Una versión algo abreviada de esta obra se tradujo al francés (Histoire religieuse de l'Espagne, Cerf, Paris, 1998) dentro de la reconocida serie «L'Histoire religieuse de l'Europe contemporaine».

${ }^{23}$ La actas del coloquio de la Casa de Velázquez en Benoit Pellistrandi (ed.), L'histoire religieuse en France et en Espagne, Casa de Velázquez, Madrid, 2004. 
tituciones ${ }^{24}$. Desde luego la amenaza y la movilización secularizadora no era comparable en España a la situación francesa o italiana, y no se planteó y percibió de forma clara hasta la crisis de 1898; además la sólida posición institucional de la Iglesia, en el marco de los vigentes Concordato de 1851 y la Constitución de 1876, apenas hacían necesario el uso del Movimiento Católico como contraofensiva. De modo que en comparación con el italiano, el Movimiento católico español era en cierta medida más débil, y tenía una configuración y un contenido más tradicional, de acuerdo con el peso en el catolicismo español de la «tesis» integrista sobre la «hipótesis» posibilista. Todo ello, junto a una primera consulta detenida de materiales en el Archivo Vaticano sobre el tiempo de León XIII y Pío X, inspiró la síntesis breve y sin notas (por exigencias editoriales) del Movimiento Católico en España, desde el principio necesitada de una ampliación ${ }^{25}$.

El Modernismo religioso y la crisis modernista de principios del siglo XX era un momento clave para la comprensión del conflicto catolicismo-laicismo. En este sentido el temprano encuentro e intercambio intelectual y amistoso con el hispanista italiano Alfonso Botti resultó fundamental para el impulso de los proyectos personales y colectivos de la nueva historia religiosa española. Su participación activa en cursos y publicaciones organizados en el entorno de nuestros proyectosI+D ha contribuido a estimular la investigación española, liberándola de algunos complejos y obstáculos tradicionales, animando y orientando siempre la perspectiva comparada con la historiografía italiana ${ }^{26}$.

24 Cfr. Feliciano Montero, El primer catolicismo social y la Rerum Novarum 1889-1902, CSIC, Madrid, 1983. Otros autores, como José Andrés-Gallego, Cristóbal Robles o Andrés Martínez Esteban, se han referido en sus respectivos trabajos a estos Congresos, pero aun falta un estudio sistemático de los mismos.

${ }_{25}$ Feliciano Montero, El Movimiento Católico en España, Eudema, Madrid, 1993 Muy pronto me planteé una edición ampliada y con notas, a partir de los diversos materiales acumulados. En esta época C. Robles publicó mucha información en una serie de artículos en Antologica Annua, bajo la rúbrica, «Frente a la supremacía del Estado: la Santa Sede y los católicos de la Restauración», Antologica Annua, 34, 1987,pp. 189-306. Posteriormente su discípulo Andrés Martínez Esteban elaboró su tesis en Roma, Aceptar el poder constituido. Los católicos españoles y la Santa Sede en la Restauración, 1890-1914, San Dámaso, Madrid, 2006.

26 Alfonso Botti,_La Spagna e la crisi modernista, Morcelliana, Brescia, 1987. Sus contribuciones a nuestros proyectos I+D van más allá de su participación en las publicaciones. Por otra parte, no hace falta recordar su influencia en general en la historiografía española sobre el siglo Xx, a través de la revista Spagna Contemporanea y de los encuentros historiográficos hispano-italianos celebrados en Novi. 


\section{Del Movimiento Católico a la Acción Católica}

Algunos estudios sobre la historia de la Acción Católica española, comenzando por el análisis de una coyuntura específica y reveladora, la llamada «crisis de la Acción Católica» de 1966-68, me acercaron también a la historia del «tiempo presente». Pues en este caso la investigación en la documentación y en los archivos de la Acción Católica española se cruzaba «peligrosamente» con el recuerdo y el testimonio personal. Las preguntas historiográficas sobre los retos y acomodos del catolicismo español en el siglo XX, desde la «cuestión social» hasta el laicismo, cobraban nueva dimensión en el estudio de la evolución y posición de la Acción Católica especializada por ambientes sociales, especialmente la juvenil ${ }^{27}$, frente a un régimen político autoritario y una Iglesia vinculada y «protegida», centrada en una pastoral de cristiandad. Partiendo pues de este acercamiento primero al estudio de la Acción Católica en los años 1960 y la crisis de 1966-68, traté de reconstruir la historia de la Acción Católica, propiamente dicha, según el modelo impulsado por Pío XI en el periodo de entreguerras, desde sus antecedentes anteriores a la guerra civil hasta su época de esplendor durante el franquismo; distinguiendo tiempos, como el relevante, aunque corto, de la II República, o los correspondientes al primer y segundo franquismo ${ }^{28}$. Ya en la breve síntesis de El Movimiento Católico se esbozó la diferencia entre el conjunto del Movimiento Católico, en su triple dimensión propagandística, social y política, y la organización esencialmente apostólica (dependiente de la Jerarquía) y apolítica (o más bien prepolítica) de la Acción Católica.

27 Uno de los temas centrales fue el estudio de la conversión de los centros parroquiales de la JACE en Movimientos especializados por ambientes, obrero (JOC, campesino (JARC) estudiantil (JEC), urbano (JIC), en los cinco primeros años de 1960; y de la celebración de una Campaña Conjunta y Asamblea Nacional en el verano de 1965, sobre la Participación de la Juventud en los distintos ámbitos sociales. El libro La Acción Católica en el franquismo. Auge y crisis de la AC especializada, UNED, Madrid, 2000, es una síntesis de la evolución del conjunto de la AC, no sólo la obrera o la juvenil, en los años 1960, y de la crisis o conflicto con la Jerarquía en los años 1966-68.

${ }^{28}$ El estudio de la AC española durante la II República fue objeto de un seminario de un proyecto I+D, cfr. Feliciano Montero (ed.) La Acción Católica en la II República, Universidad de Alcalá, Alcalá de Henares, 2008. Me queda pendiente una síntesis de la historia de la AC española a partir de los diversos trabajos monográficos publicados. 
Una vez más el estudio de la Acción Católica española, como anteriormente el del catolicismo social y el Movimiento católico, remitía al análisis de las influencias y relaciones internacionales en una perspectiva transnacional. Ya en los viajes de los consiliarios de la AC, durante los veranos de 1933 y 1934, se apreciaba la doble dependencia del modelo italiano (AC parroquial) y franco-belga (AC especializada), que se prolonga en el tiempo del franquismo. De ahí la importancia de la perspectiva comparada con la historiografía francesa, portuguesa y, en este tema, sobre todo italiana ${ }^{29}$.

La Acción Católica especializada como plataforma de conciencia política, ciudadana, democrática y, por tanto, antifranquista, era una vía para adentrarse en el estudio del factor católico en el proceso de la Transición, o más bien de la preparación (la pretransición), considerando distintas instancias además de la AC, y sus antecedentes más remotos, incluida la «autocrítica religiosa» de principios de los años $1950^{30}$.

\section{La historiografía portuguesa}

Los contactos, más recientes, con la historiografía religiosa portuguesa, en el marco del Centro de Estudios de Historia religiosa de la Universidad Católica Portuguesa, han ampliado y reforzado la necesaria perspectiva transnacional de nuestros estudios sobre el conflicto catolicismo-laicismo en la España del siglo XX. Han significado el descubrimiento de una realidad histórica más análoga, y de una historiografía renovada y muy activa. Las primeras aproximaciones a la historia portu-

${ }^{29}$ Especialmente abundante la historiografía italiana de acuerdo con la relevancia institucional y orgánica de la AC italiana en la Iglesia y el catolicismo italiano; por otra parte, mucho más resistente, aunque no inmune, a las crisis de los años 1950 y 1960, que la AC francesa o la española. Diversos estudios de Mario Casella, L' azione Cattolica nell'Italia contemporanea (1919-1969), AVE, Roma, 1992 o Ernesto Preziosi, Piccola storia di una grande associazione. L'Azione Católica in Italia, AVE, Roma, 2002. Sobre la Acción Católica portuguesa, Paulo Fontes, Elites católicas em Portugal: o papel da Acção Católica Portuguesa (1940-1961), Universidade Católica de Lisboa, Lisboa, 2006.

30 Diversos trabajos sobre este tema, comenzando por el articulo «Iglesia y transición» en el numero monográfico sobre la Transición de Ayer, 15 (1984), culminaron en la síntesis Feliciano Montero, La Iglesia: de la colaboración a la disidencia, Encuentro, Madrid, 2009. 
guesa revelaban las analogías en los procesos y en las cuestiones históricas, independientemente de las escasas relaciones directas, y del desconocimiento inicial recíproco; y, por tanto, la potencialidad de esos estudios comparados y de los encuentros. Por otra parte, la dependencia común de la historiografía francesa y de una mentalidad (cultura) compartida en organizaciones internacionales católicas (JEC, Pax Romana) facilitaba el encuentro y el diálogo de los investigadores ${ }^{31}$.

Como ya señaló Javier Tusell en sus reflexiones sobre la naturaleza fascista o autoritaria del régimen de Franco, la comparación con el régimen de Salazar, apenas estudiada entonces y aún hoy poco explorada, podía ser más fructífera que la más transitada historiográficamente con el fascismo italiano ${ }^{32}$. Esto mismo se puede aplicar al estudio de los respectivos catolicismos y de su relación de colaboración (identificación) y posterior distanciamiento con los respectivos regímenes autoritarios. Sin olvidar, por otra parte, diferencias históricas relevantes como la pervivencia del régimen de Separación de 1910, con todas las modificaciones, durante el salazarismo, a diferencia de la estricta confesionalidad proclamada en el Concordato español de 1953, que se prolonga hasta la Constitución de la Transición de 1978.

\section{Catolicismo y laicismo en la España del siglo $\mathrm{xx}$}

En los primeros estudios sobre el catolicismo social y la recepción de la Rerum Novarum (1983), y más claramente en la breve síntesis sobre la historia del Movimiento católico (1993) ya subyacía el estudio de la confrontación catolicismo-laicismo en torno al rechazo-adaptación-asunción de la modernidad. Pero fue a partir de la comunicación sobre «Clerica-

${ }^{31}$ Es el caso de dos de los principales investigadores del Centro de Estudos de História Religiosa de la Universidade Católica de Lisboa, Antonio Matos Ferreira y Paulo Fontes, relacionados en su juventud con Pax Romana y con la JEC. La participación recíproca en encuentros y cursos en Alcalá y especialmente en Lisboa han posibilitado un conocimiento y comparación, que está por desarrollar. Véase especialmente la participación española en trabajos colectivos como el Antonio Matos Ferreira y Joâo Miguel Almeida (coords.), Religião e cidadania: protagonistas, motivações e dinâmicas sociais no contexto ibérico, Centro de Estudos de História Religiosa/ Universidade Católica Portuguesa, Lisboa, 2011 (con trabajos de Cristóbal Robles, José Ramón Rodríguez Lago y Laura Serrano).

32 Javier Tusell, La dictadura de Franco, Alianza, Madrid, 1988. 
lismo y anticlericalismo en torno a la crisis de 1898», presentada conjuntamente con Julio de la Cueva en el Congreso de la Asociación de Historia Contemporánea de 1998 en Sevilla, cuando comenzamos a perfilar el Proyecto de Investigación en torno al cual se fue configurando un equipo de investigadores y una serie de actividades y publicaciones. A partir de este momento se puede hablar más de una trayectoria colectiva que personal, y de un trabajo de coordinación y de síntesis que de investigación monográfica.

El eje argumental y los presupuestos de los sucesivos Proyectos I+D que nos han sido concedidos a partir del 2002 estaban ya planteados en la comunicación conjunta de 1998 . Se trataba de estudiar conjunta y recíprocamente los dos polos del conflicto o confrontación, los dos movimientos y culturas, definidas «positivamente», movimiento católico (recristianizador) versus movimiento secularizador. Dos polos bien definidos pero no monolíticos, en los que se debían reconocer matices y tendencias. Una confrontación global, total, pero que debía ser analizada en sus diversas expresiones y dimensiones: no sólo la más conocida, la ideológica, doctrinal o propagandística, sino su plasmación política y social en el terreno educativo, en el asistencial, en los modos de vida y moralidades y comportamientos. La composición del equipo y la selección de ponentes en los seminarios y publicaciones, además de cubrir esas dimensiones, pretendía superar la habitual reproducción historiográfica - recíprocamente excluyente- de la confrontación histórico-política ${ }^{33}$.

\section{Conclusiones}

La trayectoria historiográfica que he recordado ha estado centrada principalmente en la historia del catolicismo social y político español, en el marco latino más próximo, a lo largo del siglo Xx, (arrancando de la Restauración canovista de 1876). De modo que sólo parcialmente

${ }^{33}$ Una presentación de esta línea y de Proyectos I+D en Feliciano Montero, «Catolicismo y laicismo en la España de la Restauración (en el marco europeo mediterráneo)» en José Manuel Delgado Idarreta, Julio Pérez Serrano y Rebeca Viguera Ruiz (eds.), Iglesia y Estado en la sociedad actual. Política, Cine y Religión, I. Estudios Riojanos, Logroño, 2014, pp. 53-75. Una valoración externa de nuestros proyectos en Pilar Salomón, «Libertad religiosa y laicismo en la España contemporánea. Reflexiones sobre algunas perspectivas historiográficas recientes», Ayer, 86 (2012) pp. 227-245 
se puede presentar como una historia metodológicamente renovada, de acuerdo con el programa de la historiografía francesa de los años 1970 y 80 que evocábamos al principio. Ese horizonte de nueva historia religiosa, social y cultural, ha sido más una referencia a impulsar y orientar que una práctica propia.

Desgraciadamente la historia religiosa social, según el proyecto francés de historias diocesanas, se planteó excepcionalmente, como se ha mencionado, en el estudio de Antón Pazos sobre el clero navarro, o el más reciente de José Luis González Gullón sobre el clero de Madrid en los años 1930, o en algunos estudios de diócesis y de obispos, que se han planteado como algo más que una mera biografía ${ }^{34}$. Algunos de los apartados de la síntesis de José Andrés-Gallego y Antón Pazos sobre la Iglesia española presentan bien el programa de trabajo, pero la investigación, especialmente la encuesta sociológica retrospectiva, tanto en el plano nacional como en el diocesano, queda aún por hacer; y no solamente por ausencia de fuentes, en todo caso quizá por su escaso inventario y difícil acceso $^{35}$.

La alarma sobre la «apostasía de las masas» y su consiguiente reto pastoral suscitó algunas encuestas sociológicas sobre la práctica religiosa y en general sobre la supuesta o real descristianización y sus causas, que estimularon algunas investigaciones históricas. Mucho menos intensas que las llevadas a cabo por la sociología religiosa pastoral en los años 1960 , siguiendo los pasos de la sociología francesa ${ }^{36}$.

${ }^{34}$ Un ejemplo de ello pueden ser las ya citadas obras Casimir Martí sobre el obispo Costa i Borras, de C. Ibañez sobre Vilatmijana en Tortosa y de Jordi Figuerola sobre Morgades en Vich. Más recientemente, también podemos destacar los trabajos de Santiago Martínez, Los papeles perdidos del Cardenal Segura, 1880- 1957, Eunsa, Pamplona, 2004 y Miguel Ángel Dionisio, Isidro Gomá ante la Dictadura y la República, Instituto Teológico San Ildefonso, Toledo, 2011.

35 Aquí queda pendiente una mejor y mayor comunicación con la Asociación Española de Archiveros y Bibliotecarios Eclesiásticos que, por otra parte, viene desarrollando desde hace tiempo (fue fundada en 1971) una buena labor. Para profundizar en su labor pueden consultarse la revista Memoria Ecclesiae, que se edita de forma regular desde 1988 y que recoge las actas de los sucesivos congresos que organizan.

36 Sobre la respuesta pastoral en los años 1930 a la «apostasía de las masas», siguiendo reflexiones de los propios protagonistas como Arboleya, Azpiazu, Peiró, vid. Feliciano Montero, «La apostasía de las masas y la recristianización de la sociedad: las estrategias pastorales de la Iglesia española en el siglo XX» en El siglo XX: balance y perspectivas, Publicacions de la Universitat de Valencia,Valencia, 2000, pp. 391-398. Por otro lado, la muestra mejor de la sociología religiosa pastoral en España, R. Duocastella, Analisis socio- 
Si el impacto y el desarrollo de la historia social religiosa en la historiografía española ha sido relativamente escaso y débil, y sus objetivos quedan todavía por cumplir, la nueva historia cultural, antropológica y social, sí está teniendo más influencia en la nueva historia religiosa. Desde una perspectiva eminentemente teológica, litúrgica y pastoral, Álvarez Bolado puso las bases de una lectura diferente del discurso pastoral de la Iglesia sobre la guerra civil, acompañando en el tiempo litúrgico las vicisitudes de la guerra, y su vivencia y experiencia ${ }^{37}$. Más claramente se puede apreciar en el enfoque general de la tesis doctoral de Joseba Louzao sobre el conflicto político-religioso en Vizcaya, durante la Restauración, y en otras investigaciones del mismo autor ${ }^{38}$. Pero también en los estudios sobre devociones y cultos, como los trabajos pioneros de Giuliana di Febo sobre los cultos del franquismo, la tesis de Francisco Ramón Solans sobre la devoción a la Virgen del Pilar, o los estudios coordinados por Rafael Serrano sobre el siglo XIX ${ }^{39}$. En esta misma línea, el estudio de algunas obras e iniciativas católicas de ocio y educación popular se han enriquecido desde la perspectiva de la nueva historia cultural ${ }^{40}$. Quizá no sea casual, y debe interpretarse como una señal del cambio de la historia

lógico del catolicismo español, Nova Terra, Barcelona,1967 (cfr. Francisco Carmona, «La sociología religiosa pastoral francesa y su influencia en España», ponencia inédita presentada en el Curso, Catolicismo, Franquismo y Oposición: Crisis y autocríticas en los años 1950, UAH, octubre 2014).

37 Alfonso Álvarez Bolado, Para ganar la guerra, para ganar la paz. Iglesia y guerra civil: 1936-1939, Universidad Pontificia Comillas, Madrid, 1995.

38 Joseba Louzao, Soldados de fe o amantes del progreso: Catolicismo y modernidad en Vizcaya (1890-1923), Genueve, Madrid, 2011, Joseba Louzao, «La recomposición religiosa en la modernidad: un marco conceptual para comprender el enfrentamiento entre laicidad y confesionalidad en la España contemporánea», Hispania Sacra, 121 (2008), pp. 331-354 o Joseba Louzao, «Catholicism Versus Laicism: Culture Wars and the Making of Catholic National Identity in Spain, 1898-1931», European History Quarterly, 43 (2013), pp. 657-680.

39 Giuliana Di Febo, Ritos de guerra y de victoria en la España franquista, Desclée de Brouwer, Bilbao 2002; Francisco Javier Ramón Solans, La Virgen del Pilar dice... Usos políticos y nacionales de un culto mariano en la España contemporánea, Prensas Universitarias de Zaragoza, Zaragoza, 2014 o Rafael Serrano García, Angel de Prado Moura y Elisabel Larriba (coords.), Discursos y devociones religiosas en la Península Ibérica, 1780-1860: de la crisis del Antiguo Régimen a la consolidación del Liberalismo, Universidad de Valladolid, Valladolid, 2014

40 Francisco Javier Caspistegui, «Religión, tradicionalismo y espectáculos de masas» en Jaume Aurell y Pablo Pérez López (eds.), Católicos entre dos guerras: la historia religiosa de España en los años 20 y 30, Biblioteca Nueva, Madrid, 2006, pp. 327-349. 
eclesiástica a la historia religiosa, que en el último número de la revista Ayer de 2014 (el número 96), dedicado a estudios premiados de jóvenes investigadores, varios trabajos se situaban en el ámbito de la historia de la cultura religiosa católica ${ }^{41}$. El futuro es prometedor.

${ }^{41}$ Raúl Mínguez, «Las múltiples caras de la Inmaculada: religión, género y nación en su proclamación dogmática (1854)» (pp. 39-60) y Francisco Javier Ramón Solans, «Milagros, visiones apocalípticas y profecías. Una lectura sobrenatural de la Guerra de la Independencia» (pp. 83-104). 